\title{
Wind-integration benefits of controlled plug-in electric vehicle charging
}

\author{
Sachin CHANDRASHEKAR ${ }^{1}$, Yixian LIU $^{1}$, Ramteen SIOSHANSI $^{1}$ (i)
}

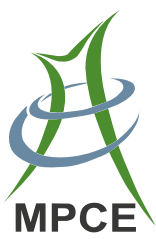

\begin{abstract}
Flexibility in plug-in electric vehicle (PEV) charging can reduce the ancillary cost effects of wind variability and uncertainty on electric power systems. In this paper, we study these benefits of PEV charging, demonstrating that controlled PEV charging can reduce costs associated with wind uncertainty and variability. Interestingly, we show that the system does not require complete control of PEV-charging loads to mitigate the negative cost impacts of wind variability and uncertainty. Rather, PEV owners giving the system a two-hour window of flexibility in which to recharge their vehicles provides much of the benefits that giving full charging control does.
\end{abstract}

Keywords Plug-in electric vehicle, Controlled charging, Wind integration, Demand response, Unit commitment and dispatch

\section{Introduction}

Concerns surrounding growing energy demand, climate change, and finite fossil-fuel supplies have increased interest in the use of renewable energy resources.

CrossCheck date: 22 March 2017

Received: 14 May 2016/Accepted: 22 March 2017/Published online: 8 September 2017

(C) The Author(s) 2017. This article is an open access publication

$\triangle$ Ramteen SIOSHANSI

sioshansi.1@osu.edu

Sachin CHANDRASHEKAR

chandrashekar.15@osu.edu

Yixian LIU

liu.2441@osu.edu

1 Department of Integrated Systems Engineering, The Ohio State University, Columbus, OH, USA
Renewables, such as wind, can significantly reduce electricity-related emissions and costs by displacing conventional fossil-fueled resources. They can, however, have ancillary cost impacts on other generators. This is because their real-time output is uncertain and variable when making operational decisions day- or even hour-ahead. More specifically, to accommodate this variability, conventional generators may have to frequently adjust their output levels or be cycled on and off. A power system may also need more reserves from conventional generators to balance wind uncertainty, forcing generators to operate inefficiently in a partially loaded fashion $[1,2]$. Empirical numerical studies of the Belgian [3], Irish [4], Texas [5], and U.K. [6] power systems further demonstrate these effects of wind.

One commonly proposed means of mitigating these cost impacts of renewables is to use some form of demand response, whereby flexible demands follow the availability of wind. Through such a scheme, demand responds to unexpected drops in the availability of wind, reducing the need to use costly conventional generators to replace renewable supply. Demand can also respond to unexpected increases in wind availability, reducing possible wind curtailment due to generator or transmission constraints [7]. A number of numerical studies [5, 8, 9] show that demand response can effectively reduce the ancillary costs of wind uncertainty and variability.

A limitation of these analyses is that they do not answer the question of what exact loads respond to wind availability. Instead they assume that some portion of the load is flexible and responds to price or other signals indicating wind availability. A natural source of load flexibility is plug-in electric vehicle (PEV) charging. A PEV owner is typically only concerned with having energy recharged into the PEV's battery before the next vehicle departure. Thus, 
there is potentially flexibility in when and the rate at which a PEV is recharged between its arrival to and subsequent departure from a charging station. Properly controlled PEV charging can, therefore, provide demand response to accommodate wind variability.

A number of works examine how to control PEV charging. Reddy et al. [10] develop a fireworks algorithm to schedule PEV charging and the use of renewable energy to minimize power system emissions and cost. Liu et al. [11] develop a PEV charging-control algorithm to optimize the peak-valley difference of a power system, considering wind and solar production. Liu et al. [12] survey a variety of centralized and decentralized PEV-charging-control algorithms. They present a mathematical framework, which they propose using to evaluate the positive and negative characteristics of the algorithms in terms of different actors in the power system. Momber et al. [13] introduce a PEV-charging control model that accounts for risk-aversion.

In this paper we use a numerical case study, based on the Electricity Reliability Council of Texas (ERCOT) system, to demonstrate the benefits of controlled PEV charging on wind integration. We focus on the impacts of controllable PEV-charging loads on reducing the costs arising from wind variability and uncertainty. Our analysis assumes that PEVs are used solely to provide demand response. This means that the timing and rate of PEV charging can be adjusted based on wind availability and other power-system conditions. Our analysis does not allow PEV batteries to be discharged to provide so-called vehicle-to-grid services [14-16]. Moreover, we require each PEV to be fully recharged by the time it finishes each stop at a charging station. Thus, the demand response that we model does not allow PEV-charging load to go unserved. Rather, it only allows those loads to be shifted during the window of time that a vehicle is grid connected.

Our case study examines PEV charging and power system operations over a one-year period. We assume about $7 \mathrm{GW}$ of wind is added to a system with a peak nonPEV load of about $60 \mathrm{GW}$. We further assume that a fleet of about 50000 PEVs, which require a total of about $470 \mathrm{MWh}$ of energy to be recharged into their batteries each day, is added to the system.

We demonstrate that without the PEVs, wind uncertainty and variability impose an ancillary cost of about $\$ 0.23 / \mathrm{MWh}$ of wind. This cost increases to $\$ 0.46 / \mathrm{MWh}$ of wind if PEV charging is not controlled (i.e., PEVs charge immediately upon arrival at a charging station). However, if PEV charging can be fully controlled, the ancillary cost of wind uncertainty and variability is reduced to $\$ 0.09 / \mathrm{MWh}$. We also examine a case in which there is a limited two-hour window of flexibility within which PEV charging can be controlled. We show that such a two-hour window of flexibility provides much of the benefits of complete PEV-charging control. This is because the benefit of controlled PEV charging is in allowing the system to more easily accommodate errors in estimating wind availability. Although wind forecasts can be wrong in a particular hour, these errors tend to smooth out over the course of several hours. Thus, some limited flexibility in shifting PEV-charging loads around within two hours provides almost as much benefit as complete control of PEV charging in reducing wind-integration costs. The choice of studying a two-hour window of flexibility is meant to represent an intermediate case of charging control, between the extremes of uncontrolled and fully controlled PEV charging. One could study other intermediate cases, which is an area of further study.

The remainder of this paper is organized as follows. Section 2 details the models that are used to examine how the power system is operated and PEVs are recharged under the different cases constituting our study. Section 3 summarizes the data and assumptions underlying our numerical case study. Section 4 presents our results and Section 5 concludes.

\section{Models and methods}

Our analysis is based on a unit commitment and dispatch model that considers the scheduling of PEV-charging loads [16]. Our model is agnostic to who actually controls PEV charging, so long as it is co-ordinated and co-optimized with the commitment and dispatch of the power system. We give a detailed formulation of our scheduling model in Section 2.1 and then explain how the model is used in analyzing different PEV-charging and wind cases to evaluate the benefits of controlled PEV charging in Section 2.2.

\subsection{Scheduling model formulation}

Our analysis studies hourly power system operations and PEV charging over a one-year period. We conduct this analysis in a rolling-horizon fashion, which is further detailed in Section 2.3. This is done by rolling forward through each hour of the year and determining system operations and PEV charging for that hour while considering future system and PEV-charging needs. In the following, we provide the formulation of the model starting from hour $m$. Note that in the rolling-horizon technique, the value of $m$ varies from 1 to 8760 as the algorithm rolls through the hours of the year. 


\subsubsection{Model notation}

We begin by first defining model notation.

1) Index parameters and sets

Our model assumes a fixed $T$-hour optimization horizon. Although we simulate power system operations and PEV charging one hour at a time, these decisions are made taking into account future power-system and PEV-charging needs over the subsequent $T$ hours. The system is assumed to have a set, $I$, of conventional generators. An additional set of wind generators are modeled, but not included in the set, $I$.

We also assume that the PEVs are categorized according to a set, $V$, of PEV-driving profiles. The PEVs modeled are each assigned to one of the driving profiles in the set, $V$.

2) Power system parameters and data

Generators are modeled using the three-part cost structure that is standard in unit commitment models. This consists of a startup cost, $c_{i}^{S}$, which is incurred whenever generator $i$ is switched on from an offline state, a no-load $\operatorname{cost}, c_{i}^{N}$, which is incurred each hour that generator $i$ is online (regardless of its generation level), and a variable cost, $c_{i}^{V}(\cdot)$, which gives the per-hour variable cost as a function of energy produced. Although $c_{i}^{V}(\cdot)$ can presumably take any form, in our case study we restrict attention to convex piecewise-linear functions, which is standard in unit commitment modeling.

Our model includes standard constraints on generator operations. Generator $i$ must produce between $Q_{i}^{-}$and $Q_{i}^{+}$MW during hours that it is online. Otherwise, it is restricted to producing $0 \mathrm{MW}$ when offline. There are also restrictions on how much the output of each generator can increase or decrease from one hour to the next. We let $R_{i}^{-}$ and $R_{i}^{+}$denote the maximum amount by which the output of generator i can decrease and increase, respectively, from one hour to the next. Generators also have restrictions on being cycled on and off. We let $\tau_{i}^{-}$and $\tau_{i}^{+}$denote the minimum number of hours that a generator must remain offline after being shutdown and online after being started up, respectively. Finally, generators have restrictions on how much reserves they can provide. We let $\rho_{i}^{S}$ denote the maximum amount of spinning reserves that generator $i$ can provide in each hour, which can only be provided during hours that the generator is online. Similarly, $\rho_{i}^{N}$ denotes the maximum amount of non-spinning reserves that generator $i$ can provide. Non-spinning reserves can be provided by generators regardless of whether they are online or offline. In addition to the limits, $\rho_{i}^{S}$ and $\rho_{i}^{N}$, any reserves provided by a generator must satisfy its ramping and capacity constraints.
Wind generators are modeled as having zero operating cost. Moreover, we let $\bar{W}_{t}$ denote total wind generation available in hour $t$.

We let $l_{t}$ denote the hour- $t$ non-PEV load. In addition to a load-balance constraint, we also impose load-based reserve restrictions. We require that, at a minimum, a fraction, $\eta^{S}$, of the hourly load be held as spinning reserves. We similarly require that, at a minimum, a fraction, $\eta^{N}$, of the hourly load be held as non-spinning reserves.

3) PEV parameters and data

Each of the PEVs that are modeled is assigned to one of the driving profiles in the set, $V$. We let $N_{v}$ denote the number of PEVs that are assigned to driving profile $v$. We further assume that all of the PEVs that are assigned to a driving profile have the same driving patterns (i.e., arrival times to and departure times from charging stations) and battery state of charge (SOC) upon arrival to the charging station. We let $\phi_{v}^{A}$ and $\phi_{v}^{D}$ denote the arrival time to and departure time from the charging station of the PEVs that are assigned to driving profile $v$.

We let $\zeta_{v}$ denote the remaining charging energy that must be supplied to PEVs in driving profile $v$. The value of $\zeta_{v}$ is updated in our rolling-horizon algorithm ( $c f$. Section 2.3) as PEV-charging decisions are made on an hourby-hour basis. We assume that a PEV can only be charged between its arrival and departure times and that the full $\zeta_{v}$ MWh of charging demand must be supplied to each PEV in driving profile $v$ before its departure time in hour $\phi_{v}^{D}$. All PEVs are assumed to connect to the same type of charging station with a charging capacity of $\bar{H} \mathrm{MW}$.

Each PEV-driving profile is modeled as having a single arrival and departure time. In practice, a PEV may have multiple trips and be parked at a charging station multiple times during a day. We capture multiple parking events by representing each event as a separate driving profile. As an example, suppose that a PEV is parked from 9 am until $11 \mathrm{am}$, then departs the charging station, and is parked again from $3 \mathrm{pm}$ until $7 \mathrm{pm}$. This would be represented by breaking the PEV into two driving profiles. The first would have arrival and departure times at 9 am and $11 \mathrm{am}$, and the model would require the PEV to be fully recharged before its 11 am departure. The second driving profile would have $3 \mathrm{pm}$ and $7 \mathrm{pm}$ arrival and departure times.

4) Decision variables

We represent generator-commitment decisions using three sets of binary variables. The variable $u_{i, t}$ is defined to equal 1 is generator $i$ is online in hour $t$ and equal 0 otherwise. Similarly, $h_{i, t}$ and $s_{i, t}$ are defined to equal 1 if generator $i$ is shutdown and started up in hour $t$, respectively, and equal 0 otherwise. Generator-dispatch decisions are modeled using another three sets of continuous variables. We let $q_{i, t}$ denote generator $i$ 's hour- $t$ production 
level in MW. We also let $r_{i, t}^{S}$ and $r_{i, t}^{N}$ denote the MW of spinning and nonspinning reserves, respectively, provided by generator $i$ in hour $t$. We represent the dispatch of wind generators with another set of continuous variables. We let $w_{t}$ denote hour- $t$ wind production in MW.

We assume that all of the PEVs that are assigned to a driving profile have the same charging profile assigned to them. We let $z_{v, t}$ denote the MW of power recharged in hour $m$ into each PEV with driving profile $v$.

\subsubsection{Model formulation}

Our hour- $m$ scheduling model is formulated as:

$$
\begin{aligned}
\underset{h, q, r, s, u, w, z}{\min } & \sum_{t=m}^{T+m} \sum_{i \in I}\left[c_{i}^{V}\left(q_{i, t}\right)+c_{i}^{N} u_{i, t}+c_{i}^{S} s_{i, t}\right] \\
\text { s.t. } & l_{t}+\sum_{v \in V} N_{v} z_{v, t}=\sum_{i \in I} q_{i, t}+w_{t} \quad \forall t=m, \ldots, T+m
\end{aligned}
$$

$$
\begin{aligned}
& \sum_{v \in V} N_{v} z_{v, t}+\sum_{i \in I} r_{i, t}^{S} \\
& \geq \eta^{S} \cdot\left(l_{t}+\sum_{v \in V} N_{v} z_{v, t}\right) \quad \forall t=m, \ldots, T+m \\
& \sum_{v \in V} N_{v} z_{v, t}+\sum_{i \in I}\left(r_{i, t}^{S}+r_{i, t}^{N}\right) \\
& \geq\left(\eta^{S}+\eta^{N}\right) \cdot\left(l_{t}+\sum_{v \in V} N_{v} z_{v, t}\right) \quad \forall t=m, \ldots, T+m
\end{aligned}
$$

$$
Q_{i}^{-} u_{i, y} \leq q_{i, t} \quad \forall t=m, \ldots, T+m ; i \in I
$$

$q_{i, t}+r_{i, t}^{S} \leq Q_{i}^{+} u_{i, y} \quad \forall t=m, \ldots, T+m ; i \in I$

$q_{i, t}+r_{i, t}^{S}+r_{i, t}^{N} \leq Q_{i}^{+} \quad \forall t=m, \ldots, T+m ; i \in I$

$0 \leq r_{i, t}^{S} \leq \rho_{i}^{S} u_{i, t} \quad \forall t=m, \ldots, T+m ; i \in I$

$0 \leq r_{i, t}^{N} \leq \rho_{i}^{N} \quad \forall t=m, \ldots, T+m ; i \in I$

$R_{i}^{-} \leq q_{i, t}-q_{i, t-1} \quad \forall t=m, \ldots, T+m ; i \in I$

$q_{i, t}+r_{i, t}^{S}+r_{i, t}^{N}-q_{i, t-1} \leq R_{i}^{+} \quad \forall t=m, \ldots, T+m ; i \in I$

$\sum_{\xi=t-\tau_{i}^{+}}^{t} s_{i, \xi} \leq u_{i, t} \quad \forall t=m, \ldots, T+m ; i \in I$

$1-\sum_{\xi=t-\tau_{i}^{i}}^{t} h_{i, \xi} \geq u_{i, t} \quad \forall t=m, \ldots, T+m ; i \in I$

$u_{i, t}-u_{i, t-1}=s_{i, t}-h_{i, t} \quad \forall t=m, \ldots, T+m ; i \in I$

$$
\begin{aligned}
& u_{i, t}, s_{i, t}, h_{i, t} \in\{0,1\} \quad \forall t=m, \ldots, T+m ; i \in I \\
& 0 \leq w_{t} \leq \bar{W}_{t} \quad \forall t=m, \ldots, T+m \\
& 0 \leq z_{v, t} \leq \bar{H} \quad \forall t=m, \ldots, T+m ; v \in V \\
& z_{v, t}=0 \quad \forall v \in V ; t \notin\left[\phi_{v}^{A}, \phi_{v}^{D}\right] \\
& \sum_{t=m}^{T+m} z_{v, t}=\zeta_{v} \quad \forall v \in V
\end{aligned}
$$

Objective function (1) minimizes the total unit commitment and dispatch cost over the $T$-hour model horizon. Our model includes three system-wide constraints. First, constraints (2) impose the hourly load-balance requirements that the sum of non-PEV and PEV-charging demand exactly equals energy produced by conventional and wind generators. Constraints (3) and (4) impose contingencyreserve requirements. We model two types of contingency reserves: spinning and non-spinning reserves. These constraints allow the reserve requirements to be met using PEV-charging loads. The reason for this is that if a system contingency (e.g., a major generator or transmission failure) occurs in real-time, PEV-charging loads can be reduced to help mitigate the supply shortfall [16]. Constraints (4) allow spinning reserves to serve the nonspinning reserve requirement. This is because spinning reserves are 'higher-quality' in the sense that they must be provided by generators that are online and able to respond to a system contingency quickly. Nonspinning reserves, conversely, can be provided by generators that are offline (so long as they are able to startup and begin producing energy within a relatively short window of time, which is reflected in whether a generator has a non-zero value for $\rho_{i}^{N}$ ).

Constraints (5)-(7) impose the capacity constraints on the conventional generators. Note that when a generator is offline (i.e., $u_{i, t}=0$ ) it is restricted to providing zero generation and spinning reserves but can provide nonspinning reserves. Otherwise, if a generator is online, it can provide any combination of energy and reserves, so long as the maximum generation level is not violated. Constraints (8) and (9) impose the reserve-capability restrictions on the conventional generators. A conventional generator can only provide spinning reserves in a given hour if it is online during that hour (hence, the right-hand sides of constraints (8) have $u_{i, t}$ terms). Generators that are qualified to provide nonspinning reserves can provide them when offline. Constraints (10) and (11) impose the ramping restrictions. As with generator upper-capacity constraints (6) and (7), ramp-up constraints (11) consider energy and reserves provided by generators in each hour. Constraints (12) and (13) impose the minimum up- and down-time restrictions when a generator is started up- or 
shutdown. Constraints (14) impose the state-transition logic by defining the values of the $s_{i, t}$ and $h_{i, t}$ variables in terms of changes in the $u_{i, t}$ variables. Constraints (15) force the state variables to take on binary values. Constraints (16) impose the limits on wind generation.

Constraints (17) impose the charger capacity, by restricting the amount recharged into each PEV based on the capacity of the charger. Constraints (18) impose the driving pattern, by only allowing each PEV to be recharged while it is parked (i.e., between hours $\phi_{v}^{A}$ and $\phi_{v}^{D}$ ). Finally, constraints (19) force each PEV to be fully recharged before leaving its charging station.

\subsection{Cases examined}

We examine the impacts of wind and PEV charging under the eight cases that are summarized in Table 1. These cases vary in how wind and PEVs are modeled. Cases 1, 3, 5, and 7 assume that future wind availability is known with perfect foresight when solving the scheduling model. This means that the values of $\bar{W}_{m}, \ldots, \bar{W}_{m+T}$ that are used in the hour- $m$ scheduling model all reflect the actual wind that will be available in each of hours $m$ through $(m+T)$. Cases $2,4,6$, and 8, conversely, assume that forecasts of future wind availability must be used when solving the scheduling model. These cases all assume that the value of $\bar{W}_{m}$ that is used in the hour- $m$ scheduling model reflects the actual wind available in hour $m$. The values of $\bar{W}_{m+1}, \ldots, \bar{W}_{m+T}$ that are used in the hour- $m$ scheduling model reflect hour- $m$ forecasts of wind availability in hours $(m+1)$ through $(m+T)$, however. Further details on how wind availability is simulated and wind forecasts are generated are given in Section 3.3.

Cases 1, 3, 5, and 7 represent counterfactuals in which future wind availability is known with perfect foresight. The cost differences between each of Cases 1, 3, 5, and 7 and each of Cases 2, 4, 6, and 8, respectively, measure the incremental cost impacts of having to schedule generation and PEV charging without perfect foresight of future wind availability. This is a commonly used metric to measure the ancillary operational cost associated with wind uncertainty

Table 1 Cases examined

\begin{tabular}{lll}
\hline PEV & Wind & \\
\cline { 2 - 3 } & Perfect foresight & Forecast \\
\hline None & 1 & 2 \\
No control & 3 & 4 \\
Two-hour control & 5 & 6 \\
Full control & 7 & 8 \\
\hline
\end{tabular}

and variability $[5,17-22]$ and is the metric that we use in our analysis.

Cases 1 and 2 assume that there are no PEVs in the system whereas Cases 3 through 8 assume that there are PEVs employing different charging strategies. Cases 3 and 4 assume that there is no control over PEV charging. In these cases, each PEV is assumed to begin charging immediately upon arriving to its charging station. These cases are modeled by fixing the values of the $z_{v, t}$ variables to

$$
z_{v, t}=\min \left\{\left(\zeta_{v}-\sum_{\xi<t} z_{v, \xi}\right)^{+}, \bar{H}\right\} \quad \forall t=\phi_{v}^{A}, \ldots, \phi_{v}^{D}
$$

in the scheduling model. Equation (20) defines the hour- $t$ uncontrolled charging demand of PEVs with driving profile $v$ as the minimum between the amount of unserved charging demand, which is defined as

$$
\left(\zeta_{v}-\sum_{\xi<t} z_{v, \xi}\right)^{+}
$$

and the charger capacity, which is $\bar{H}$.

Cases 5 and 6 assume that there is limited (two-hour) control over PEV charging. This case is modeled by first determining in which hour PEVs assigned to each driving profile will be recharged in the no-control case as:

$e_{v}=\phi_{v}^{A}+\left\lceil\frac{\zeta_{v}}{\bar{H}}\right\rceil \quad \forall v \in V$

where $\lceil\cdot\rceil$ is the ceiling operator. Equation (21) defines the amount of time that it takes PEVs to recharge as the ceiling of the ratio between $\zeta_{v}$ (the amount of energy that must be recharged into the PEVs that are assigned to driving profile $v$ ) and $\bar{H}$ (the capacity of the charger). The two-hour control case assumes that PEV owners allow an additional two hours of flexibility in being fully recharged beyond the time (i.e., the end of hour $e_{v}$ ) that their PEVs would be recharged if uncontrolled. These cases are modeled by changing constraints (18) in the scheduling model to:

$$
z_{v, t}=0 \quad \forall v \in V ; t \notin\left[\phi_{v}^{A}, \min \left(\phi_{v}^{D}, e_{v}+2\right)\right]
$$

Finally, Cases 7 and 8 allow full flexibility in recharging the PEV within the window of time that it is parked at the charging station. These cases are modeled using the scheduling model that is outlined in Section 2.1, without any changes to the constraints or fixing of variables.

Figure 1 illustrates the charging windows in the three charging-control cases studied. The horizontal axis of the figure represents time and shows the case of a vehicle that is parked in the charging station between hours $\phi_{v}^{A}$ and $\phi_{v}^{D}$. In the full-control case, the scheduling model has the 


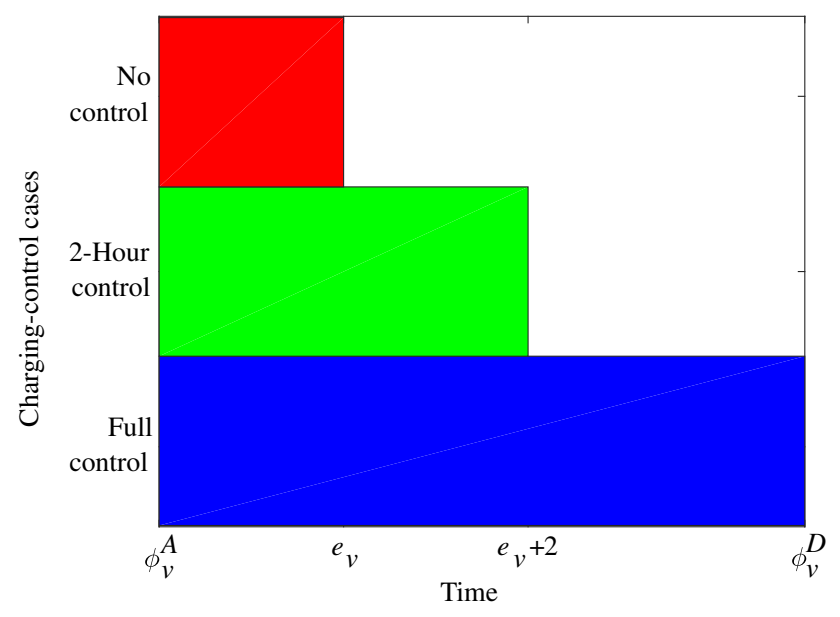

Fig. 1 Illustration of charging window in no-, two-hour-, and fullcontrol cases

flexibility to charge the PEV anytime between these arrival and departure times (so long as the vehicle is fully recharged before hour $\phi_{v}^{D}$, as constraints (19) require). In the no-control case, the PEV begins charging at the $\bar{H} \mathrm{MW}$ capacity of the charger immediately and finishes charging allowing flexibility in recharging PEVs. The choice of studying PEV charging in cases with no control, full control, and with a two-hour window of flexibility is meant to represent two extreme and one intermediate cases. One could study other intermediate cases (i.e., with half an hour of charging control). This is, indeed, an area of further study to understand how much charging flexibility is needed to harness the benefits of PEV charging in mitigating wind-integration costs.

\subsection{Rolling-horizon solution algorithm}

We simulate the commitment and dispatch of generators and the scheduling of PEV charging over a year-long period. Solving an integrated scheduling model over a year-long optimization horizon would be intractable. As such, we employ the rolling-horizon solution method that is outlined in Algorithm 1. This algorithm simulates the generator and PEV-charging scheduling process over the year one hour at a time, by rolling forward through the hours of the year.

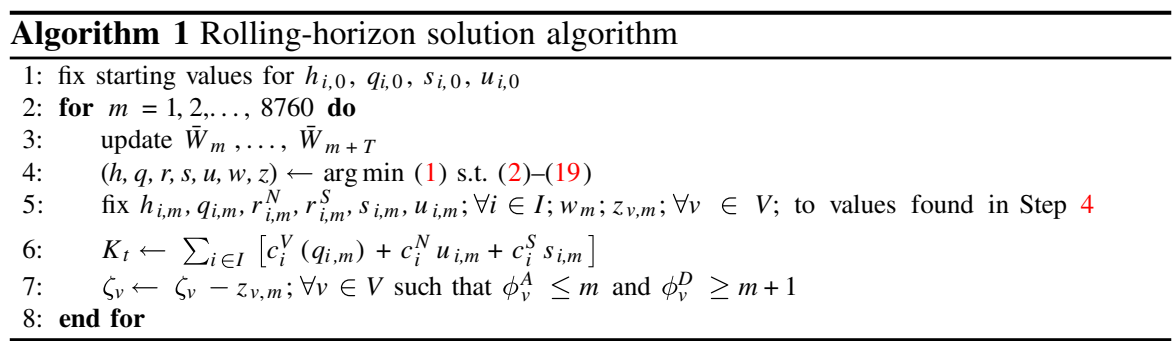

in hour $e_{v}$, as defined in (21). The two-hour-control case allows an intermediate amount of flexibility. The scheduling model can charge the vehicles within an additional two-hour window of time, relative to the no-control case. If a PEV is parked for less than two hours beyond $e_{v}$, then it is assumed to charge as in the uncontrolled case (i.e., immediately) in the two-hour-control case.

Constraints (22), defining the two-hour control case, fix values of $z_{v, t}=0$ for $t>\min \left(\phi_{v}^{D}, e_{v}+2\right)$. This is because from some driving profiles, the two-hour control window may extend beyond the period of time that the vehicle is parked. For instance, if a vehicle is parked for two hours and would require one hour of charging in the no-control case, the two-hour- and full-control cases would give the scheduling model the same window of time within which to recharge the PEV.

Cost differences between the different PEV-charging cases (i.e., between each of Cases 3 and 4, 5 and 6, and 7 and 8) allow us to gauge the cost effects and benefits of
The algorithm works by first fixing the starting state in hour 0 of all of the generators in Step 1. This is needed to set hour-1 ramping and minimum up- and down-time constraints for the generators. We assume that all of the generators are online and producing at their minimum operating points (i.e., that $q_{i, 0}=Q_{i}^{-} ; \forall i \in I$ ) and that each generator has been online a sufficient number of hours that it could be immediately switched off in hour 1, if the scheduling model finds it optimal to do so.

Steps 3 through 7 are the main iterative loop of our algorithm, which goes through each hour of the year. The loop begins in Step 3 by updating wind-availability data for the $T$ hour horizon of the hour- $m$ scheduling model. As discussed in Section 2.2 , the values of the $\bar{W}_{m+1}, \ldots, \bar{W}_{m+T}$ can either be actual wind availabilities or forecasts, depending on which of the cases that are listed in Table 1 is being modeled.

Next, Step 4 solves the hour- $m$ scheduling problem and Step 5 fixes the values of the hour- $m$ generator and PEVcharging scheduling variables only. The values of decision 
variables for hours $(m+1)$ through $(m+T)$ are determined in subsequent iterations of the algorithm. Step 6 computes the cost incurred in hour $m$, based on the values of the hour- $m$ decision variables, which are fixed in Step 5. Finally, Step 7 updates the amount of charging energy that remains unserved for vehicles that arrived before hour $m$, based on the PEV-charging decisions determined by the hour- $m$ scheduling model.

\section{Case-study data}

We study the interactions of wind and PEV charging over one year, using a case study based on the ERCOT power system. Most of our case-study data are taken from the work of Madaeni and Sioshansi [20], which is based on year-2005 data for ERCOT. We formulate the scheduling model using version 12.1.0 of the AMPL mathematical programming software package and solve it using the branch-and-cut algorithm in CPLEX 12.5.1.0 with default settings. CPLEX is an industry-standard optimization solver that is widely used in the electric-power industry.

We detail all of the data sources that are used in our analysis in the following subsections.

\subsection{Conventional-generator data}

Cost data for conventional generators are modeled using heat rates and historical fuel and $\mathrm{SO}_{2}$-permit prices. These data are obtained from proprietary databases maintained by Platts Energy and Global Energy Decisions. Conventionalgenerator-constraint data are obtained from Global Energy Decisions. The two nuclear plants in ERCOT are modeled as must-run units that constantly operate at their nameplate capacity. In total we model 375 dispatchable generators that were installed and operational in the ERCOT system in the year 2005 .

\subsection{Non-PEV-load data}

Non-PEV loads are modeled using 15-minute metered historical ERCOT load data from the year 2005, obtained from the Public Utility Commission of Texas. Because our scheduling model is formulated using hourly time steps, each of the four 15-minute measurements corresponding to each hour are averaged together to obtain an hourly-average load.

\subsection{Wind data}

Our case study assumes that there is $7 \mathrm{GW}$ of wind installed in the system, which is approximately $10 \%$ of the peak non-PEV load of about $60 \mathrm{GW}$. Thus, we study a high-penetration scenario (relative to the year 2005), considering ERCOT did not achieve $7 \mathrm{GW}$ of wind until 2008. We simulate real-time wind availability and generate wind forecasts using a vector autoregression model that is fit to three years' publicly available data from the Western Wind Resources Dataset (WWRD) for the year 2005 [23].

The WWRD consists of modeled historical wind-generation data at 10-minute intervals for numerous sites across the western United States and is generated by 3TIER for the Western Wind and Solar Integration Study [24]. Each of the sites in the WWRD is able to support $30 \mathrm{MW}$ of wind capacity. We assume that the $7 \mathrm{GW}$ of wind are distributed among the 234 WWRD locations in ERCOT that have the highest wind capacity factors.

We convert the WWRD data, which are reported at 10minute intervals, to hourly data. We then fit a vector autoregression model [25] to the WWRD data. The fitted model is then used to simulate hourly wind generation at each of the 234 WWRD locations and to generate the windavailability forecasts that are used in Cases 2, 4, 6, and 8 .

\subsection{PEV data}

PEV driving patterns are modeled using a Monte Carlobased method to generate typical daily driving patterns. We use statistical properties of light-duty vehicle driving patterns within the United States $[26,27]$ to calibrate the simulation model.

More specifically, we assume that the number of trips each vehicle makes daily has a normal distribution with a mean of 4 and a variance of 1.33 [27]. Once we simulate the number of trips each vehicles makes daily, we determine the distances of each trip. If the vehicle makes a single trip, the trip distance is randomly generated from a Weibull distribution with a mean of 36.12 miles (we use Imperial units for distances, because they are still the standard unit of measure used in the United States) and a variance of 12.04 [26]. If the vehicle makes two daily trips, the distance of each trip is half of the distance generated from the Weibull distribution (i.e., we assume that the vehicle is used for commuting to and from a workplace). If the vehicle makes three or more trips daily, two of them (which represent commuting trips) have distances that are one third of the Weibull-distributed distance. The remaining trip distances are randomly generated using a truncated non-negative Gaussian distribution. The mean and variance of the Gaussian distribution are determined so that in aggregate, one third of the vehicle miles driven are for commuting to and from the workplace [27]. The starting and ending times of each vehicle trip are determined based on the relative weights of vehicles reported to be driving at different times of day [27]. 
Table 2 Tested characteristics of 2014 Nissan Leaf

\begin{tabular}{ll}
\hline Range & 84 miles $(135 \mathrm{~km})$ \\
Battery capacity & $24 \mathrm{kWh}(21.3 \mathrm{kWh}$ usable $)$ \\
Efficiency & $0.158 \mathrm{kWh} / \mathrm{km}$ \\
\hline
\end{tabular}

We assume that the ERCOT system has 50000 PEVs, which corresponds to $0.66 \%$ of the light-duty vehicle fleet in ERCOT in 2005. The PEVs are assumed to have the characteristics (i.e., battery capacity and energy-consumption rate) of a 2014 Nissan Leaf and to connect to $5 \mathrm{~kW}$ chargers that incur $10 \%$ energy losses in recharging the vehicle batteries. Table 2 summarizes the tested characteristics of the Nissan Leaf, which are used in our analysis. The $10 \%$ energy losses in recharging the vehicle batteries is taken into account when computing the value of $\zeta_{v}$ in Step 7 of Algorithm 1. The Monte Carlo model is used to generate 1023 typical driving profiles. The $50000 \mathrm{PEVs}$ are uniformly assigned to the 1023 driving profiles, meaning that about 49 PEVs follow each driving profile. We further assume that each PEV follows the same driving profile each day.

As noted in Section 2.1, the scheduling model represents each driving profile as having a single arrival and departure time to a charging station. Because each of the simulated driving profiles can have multiple daily trips, each driving profile is subdivided into a separate profile in the scheduling model. As such, there are 4410 PEV driving profiles in the scheduling model, corresponding to these subdivisions.

\section{Case study results}

Table 3 summarizes the total annual generation costs incurred in the eight different cases ( $c f$. Table 1) examined. These costs are computed as:

$\sum_{t=1}^{8760} K_{t}$

Table 3 Annual generation costs

\begin{tabular}{llll}
\hline PEVs & $\begin{array}{l}\text { Total generation cost } \\
\text { (\$ Million) }\end{array}$ & $\begin{array}{l}\text { Wind-integration } \\
\text { cost } \$ \text { /MWh of } \\
\text { wind) }\end{array}$ \\
\cline { 2 - 3 } & Perfect foresight & Forecast & \\
\hline None & 10934.34 & 10940.67 & 0.23 \\
No control & 10928.22 & 10940.84 & 0.46 \\
Two-hour control & 10926.64 & 10930.86 & 0.15 \\
Full control & 10928.16 & 10930.68 & 0.09 \\
\hline
\end{tabular}

where the $K_{t}$ 's are defined in Step 6 of Algorithm 1. The first two columns of the table show that without PEVs and in the three different PEV-charging control cases, costs are higher when the system must be scheduled using wind forecasts as opposed to having perfect foresight of wind. This is to be expected, and the cost difference between each pair of forecast and perfect-foresight cases measures the value of perfect wind-availability information.

The last column of Table 3 reports the cost difference between the forecast and perfect-foresight cases divided by total wind generation over the course of the year. The values in this column represent the cost of wind uncertainty and variability (which we term 'wind-integration cost' in the table) on a per-MWh basis. We find that when PEVs are added to the system but their charging cannot be controlled, they double the ancillary cost impacts of wind uncertainty. However, if PEV charging can be fully controlled, wind-integration costs are reduced by close to $61 \%$. Interestingly, having only two-hours of flexibility within which to control PEV charging reduces wind-integration costs by close to $35 \%$. This means that two hours of charging control delivers close to $60 \%$ of the benefits of complete control over PEV charging.

We note that with perfect foresight of wind, the twohour-control case achieves lower total cost than the fullcontrol case. This is not unexpected, because by default CPLEX does not solve the scheduling model to complete optimality. Rather, the branch-and-cut algorithm terminates once the optimality gap of the incumbent solution is sufficiently small. In essence, the objective function of the scheduling model is extremely 'flat' around the optimum, and there are many near-optimal solutions that are virtually identical in terms of overall cost. A similar phenomenon is observed by Sioshansi and Miller [28], who find that adding emissions constraints to a model that schedules PEV-charging loads has virtually no impact on overall system costs. This cost-related finding is further evidence of the fact that the two hours of charging control delivers most of the benefits that complete charging control does. When taking into account the fact that CPLEX finds nearoptimal solutions, the two cases are very similar in terms of total overall cost.

Examining the charging profiles in the different cases provides some insights into the different interactions between PEV charging and wind. Figure 2 contrasts the total aggregate PEV charging profile on the morning of 26 July with full and no charging control with and without perfect foresight of wind. It also shows the actual wind profile and wind forecasts in hours 11 and 14.

We first note that the PEV-charging profile with no charging control does not respond in any way to system load or wind availability. As expected, much of the PEV 


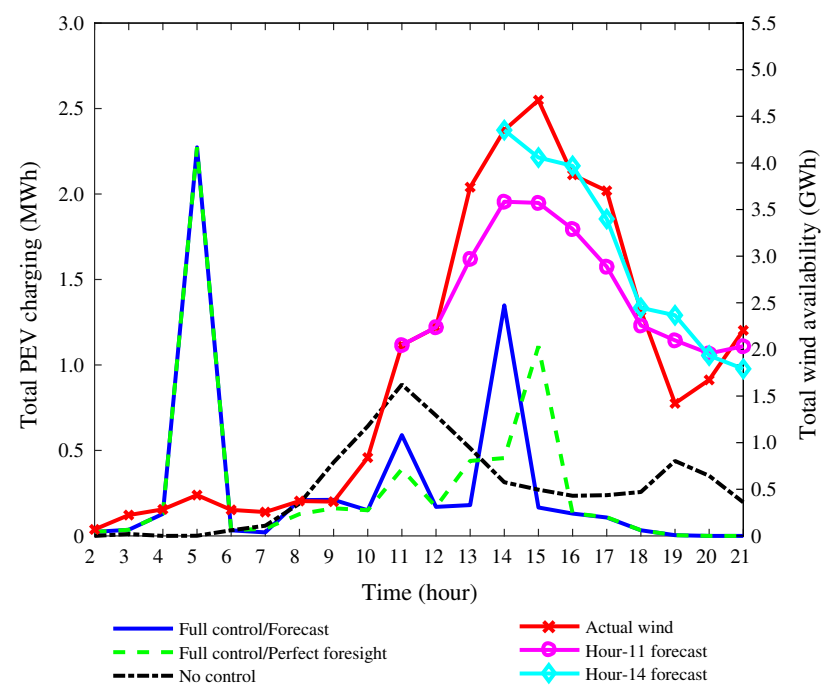

Fig. 2 Aggregate PEV-charging profiles with full control in forecasted and perfect foresight of wind cases and with no control and actual and forecasted wind on 26 July

recharging is done midday and in the early evening when vehicles first arrive at a charging station (e.g., at a workplace or stopping while running errands midday or at home in the early evening after commuting). This charging profile is undesirable because on many days the PEVs are adding load to the system when the non-PEV loads are peaking as well $[16,29]$. The PEV-charging profiles with full control, conversely, defer vehicle charging from the early evening (after commuters return home from work) to the middle of the night when the non-PEV loads and the marginal cost of generation are low.

We find that the full-charging-control cases do schedule peaks in the PEV-charging profiles midday in hours 11, 14, and 15. These peaks in the PEV-charging profiles are driven by wind availability. Contrasting the PEV-charging profiles with and without perfect foresight of wind shows the impacts of imperfect wind information. For instance, the model schedules more PEV-charging load in hours 11 and 14 when using forecasts (compared to having perfect foresight), because the model anticipates less wind being available in the future. For instance, the hour-11 forecast anticipates a total of $22.8 \mathrm{GWh}$ of wind being available between hours 11 and 18 as opposed to $27.0 \mathrm{GWh}$ actually being available. As such, the model schedules PEVcharging loads earlier than when it is optimal to do so with perfect information. Such an effect is also observed in hour 14. The hour-14 wind forecast anticipates hour 14 having the peak wind availability of the afternoon. As such, a peak in the PEV-charging load is scheduled in hour 14. In reality, however, wind availability increases in hour 15 and

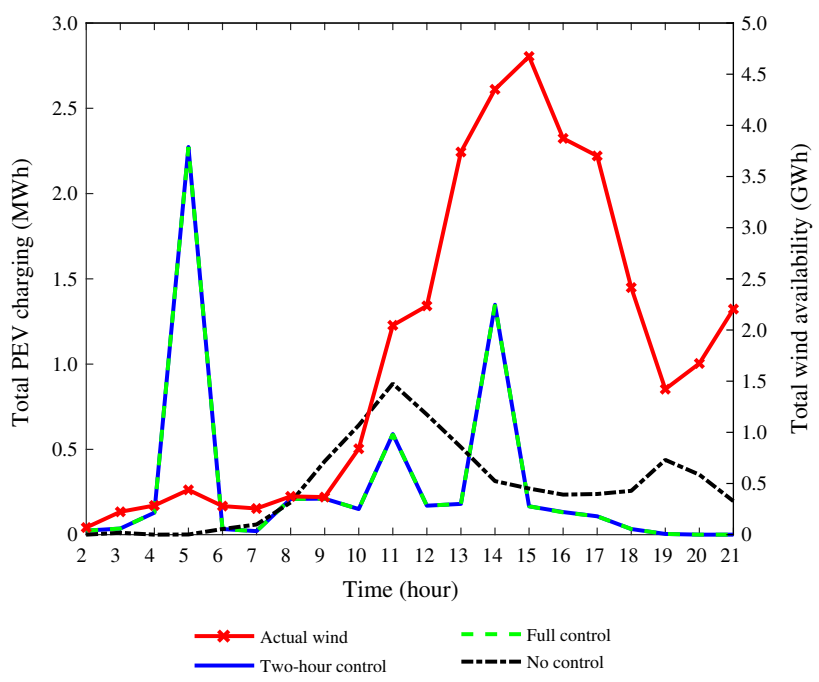

Fig. 3 Aggregate PEV-charging profiles with two-hour, full, and no control with wind forecasts used in scheduling on 26 July

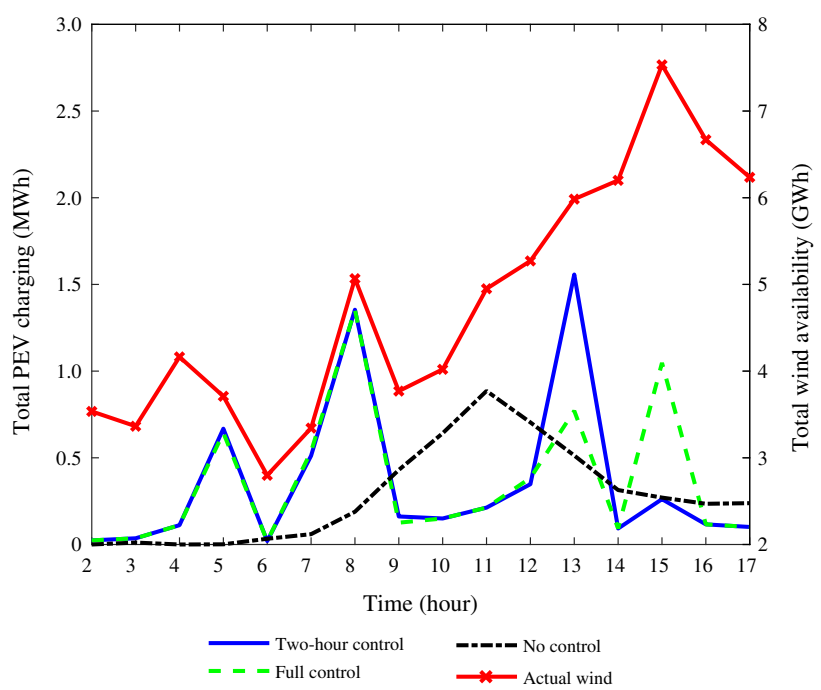

Fig. 4 Aggregate PEV-charging profiles with two-hour, full, and no control with wind forecasts used in scheduling on 24 July

delaying PEV charging an hour longer reduces system cost.

Figures 3 and 4 show two cases in which having only two-hours of charging control does not and does hamper the ability to coordinate PEV charging with power system operations. Figure 3 shows a case in which the two-hourand full-control charging profiles are virtually identical. The root mean square difference between the two charging profiles is $0.0004 \mathrm{MWh}$. The fact that the difference in the charging profiles is so small suggests that the differences may arise from numerical issues in solving the scheduling problem. 
Figure 4, conversely, shows a case in which there is a marked difference between the charging profiles in twohour- and full-control cases. Here we see that if the scheduling model has full flexibility to schedule PEV charging, it would be optimal to delay some of the vehicles to recharge in hour 15 . Doing so would, however, violate the two-hours of flexibility available in the two-hour-control case. As such, there is a larger peak in the PEVcharging profile in hour 13 , relative to the full-control case.

Although there are days, such as that shown in Figure 4, on which the two-hour limitation on control hinders the ability to properly coordinate PEV charging with power system operations, the limitation from two-hour control is relatively small. This is because the two-hour-control case achieves much of the benefits in mitigating wind-integration costs, as shown in Table 3 .

\section{Conclusion}

This paper presents an analysis of the synergies between wind and PEV charging [30]. We show that if PEV charging is not coordinated with power system operations, PEV-charging loads can exacerbate the ancillary costs of wind uncertainty and variability. This is because PEVcharging loads tend to add to peaks in the non-PEV-load profile midday and in the early evening. As such, highercost generators that are marginal in these hours must be used to balance wind availability with demand and conventional-generator supply.

Conversely, if PEV charging can be controlled, windintegration costs can be decreased substantially. Importantly, PEV owners do not have to 'hand over' full charging control to the entity operating the power system. Two hours of charging control is sufficient to achieve much of the cost savings. This is because the benefit of controlled PEV charging is in accommodating errors in estimating wind availability. Wind-forecasting errors tend to smooth out over the course of several hours. Thus, some limited flexibility in shifting PEV-charging loads around within two hours provides almost as much wind-integration benefit as complete control of PEV charging. We expect that a shorter charging window would deliver less benefits than a two-hour charging window and that a longer charging window would deliver more benefits. Our analysis shows that demand response can be used as an effective means of mitigating wind-integration costs and that PEV charging is a natural source of demand-side flexibility. Moreover, the cost savings achieved by controlling PEV charging could be used to remunerate PEV owners for making charging control available to the power system.
There are several future areas of research that can build off of this work. For one, we only examine one case of intermediate charging control in which there is a two-hour window of time within which to schedule PEV charging. A natural question is what tradeoffs are introduced by decreasing or increasing the window of time within which to control charging. This may require modeling PEV charging and power system operations at subhourly time steps. Another question is the extent to which controlling midday PEV charging is needed to mitigate wind-integration costs. This is because PEVs stopping between trips midday may be less likely to connect to a charging station (at least compared to a PEV parked overnight). A third question that we do not tackle is how to achieve the charging control assumed in our case study. The literature proposes both centralized control schemes (which would be akin to how we formulate the charging-control problem) and decentralized schemes. This is an area for further research. The work of Liu et al. [12] may give some insights into how different control schemes may fare in achieving the desired PEV-charging patterns.

Acknowledgements The authors thank Vincenzo Marano and Matteo Muratori for PEV driving-profile data. Thank you also to Armin Sorooshian, the editors, and two anonymous reviewers for helpful discussions and suggestions. The material in this paper is based upon work financially supported by the National Science Foundation (No. 1548015).

Open Access This article is distributed under the terms of the Creative Commons Attribution 4.0 International License (http:// creativecommons.org/licenses/by/4.0/), which permits unrestricted use, distribution, and reproduction in any medium, provided you give appropriate credit to the original author(s) and the source, provide a link to the Creative Commons license, and indicate if changes were made.

\section{References}

[1] Matos MA, Bessa RJ (2011) Setting the operating reserve using probabilistic wind power forecasts. IEEE Trans Power Syst 26(2):594-603

[2] Morales JM, Conejo AJ, Pérez-Ruiz J (2009) Economic valuation of reserves in power systems with high penetration of wind power. IEEE Trans Power Syst 24(2):900-910

[3] Strbac G, Shakoor A, Black M et al (2007) Impact of wind generation on the operation and development of the UK electricity systems. Electr Power Syst Res 77(9):1214-1227

[4] Denny E, O’Malley M (2006) Wind generation, power system operation, and emissions reduction. IEEE Trans Power Syst 21(1):341-347

[5] Sioshansi R (2010) Evaluating the impacts of real-time pricing on the cost and value of wind generation. IEEE Trans Power Syst 25(2):741-748

[6] Delarue ED, Luickx PJ, D'haeseleer WD (2009) The actual effect of wind power on overall electricity generation costs and $\mathrm{CO}_{2}$ emissions. Energy Convers Manag 50(6):1450-1456 
[7] Sioshansi R, Short W (2009) Evaluating the impacts of real-time pricing on the usage of wind generation. IEEE Trans Power Syst 24(2):516-524

[8] Dietrich K, Latorre JM, Olmos L et al (2012) Demand response in an isolated system with high wind integration. IEEE Trans Power Syst 27(1):20-29

[9] Klobasa M (2010) Analysis of demand response and wind integration in Germany's electricity market. IET Renew Power Gener 4(1):55-63

[10] Reddy KS, Panwar LK, Kumare R et al (2016) Distributed resource scheduling in smart grid with electric vehicle deployment using fireworks algorithm. J Mod Power Syst Clean Energy 4(2):188-199. doi:10.1007/s40565-016-0195-6

[11] Liu H, Zeng P, Guoe J et al (2015) An optimization strategy of controlled electric vehicle charging considering demand side response and regional wind and photovoltaic. J Mod Power Syst Clean Energy 3(2):232-239. doi:10.1007/s40565-015-0117-z

[12] Liu M, McNamara P, Shorten R et al (2015) Residential electrical vehicle charging strategies: the good, the bad and the ugly. J Mod Power Syst Clean Energy 3(2):190-202. doi:10.1007/ s40565-015-0122-2

[13] Momber I, Siddiqui A, Román TGS et al (2015) Risk averse scheduling by a PEV aggregator under uncertainty. IEEE Trans Power Syst 30(2):882-891

[14] Kempton W, Letendre SE (1997) Electric vehicles as a new power source for electric utilities. Transp Res Transp Environ 2(3): $157-175$

[15] Kempton W, Tomić J (2005) Vehicle-to-grid power fundamentals: calculating capacity and net revenue. J Power Sour 144(1):268-279

[16] Sioshansi R, Denholm P (2010) The value of plug-in hybrid electric vehicles as grid resources. Energy J 31(3):1-23

[17] DeMeo EA, Grant W, Milligan MR et al (2005) Wind plant integration. IEEE Power Energy Mag 3(6):38-46

[18] DeMeo EA, Jordan GA, Kalich C et al (2007) Accommodating wind's natural behavior. IEEE Power Energy Mag 5(6):59-67

[19] Fabbri A, Román TGS, Abbad JR et al (2005) Assessment of the cost associated with wind generation prediction errors in a liberalized electricity market. IEEE Trans Power Syst 20(3):1440-1446

[20] Madaeni SH, Sioshansi R (2013) Measuring the benefits of delayed price-responsive demand in reducing wind-uncertainty costs. IEEE Trans Power Syst 28(4):4118-4126

[21] Madaeni SH, Sioshansi R (2013) The impacts of stochastic programming and demand response on wind integration. Energy Syst 4(2):109-124

[22] Smith JC, Milligan MR, DeMeo EA et al (2007) Utility wind integration and operating impact state of the art. IEEE Trans Power Syst 22(3):900-908

[23] Potter CW, Lew D, McCaa J et al (2008) Creating the dataset for the Western Wind and Solar Integration Study (U.S.A.). Wind Eng 32(4):325-338
[24] Western Wind and Solar Integration Study (2010) Technical Report NREL/SR-550-47434, National Renewable Energy Laboratory

[25] Liu Y (2016) Electricity capacity investments and cost recovery with renewables. Ph.D. thesis, The Ohio State University, Columbus, Ohio, USA

[26] Pearre NS, Kempton W, Guensler RL et al (2011) Electric vehicles: How much range is required for a day's driving? Transp Res Emerg Technol 19(6):1171-1184

[27] Santos A, McGuckin N, Nakamoto HY et al (2011) Summary of travel trends: 2009 National Household Travel Survey. Technical Report FHWA-PL-11-022, U.S. Department of Transportation, Washington, DC

[28] Sioshansi R, Miller J (2011) Plug-in hybrid electric vehicles can be clean and economical in dirty power systems. Energy Policy 39(10):6151-6161

[29] Sioshansi R (2012) Modeling the impacts of electricity tariffs on plug-in hybrid electric vehicle charging, costs, and emissions. Oper Res 60(3):506-516

[30] Weiller C, Sioshansi R (2014) The role of plug-in electric vehicles with renewable resources in electricity systems. Revue d'économie industrielle 148:291-316

Sachin CHANDRASHEKAR received the B.Tech degree in chemical engineering from National Institute of Technology, Karnataka and the M.S. degree in industrial and systems engineering from The Ohio State University. His research interests focus on the integration of electric vehicles into electric power systems.

Yixian LIU received the B.E. degree in logistics engineering from Tianjin University and the M.S. and Ph.D. degrees in industrial and systems engineering from The Ohio State University. Her research interests focus on weather forecasting, electricity capacity investment, and energy-policy analysis.

Ramteen SIOSHANSI received the B.A. degree in economics and applied mathematics and the M.S. and Ph.D. degrees in industrial engineering and operations research from the University of California, Berkeley, and an M.Sc. in econometrics and mathematical economics from The London School of Economics and Political Science. He is an associate professor in the Department of Integrated Systems Engineering at The Ohio State University, Columbus, $\mathrm{OH}$. His research focuses on renewable and sustainable energy system analysis and the design of restructured competitive electricity markets. 